\title{
An Approach Toward a Feedback Mechanism for Consensus Reaching Processes Using Gamification to Increase the Experts' Experience
}

\author{
I.J. Pérez \\ University of Cádiz \\ ignaciojavier.perez@uca.es
}

\author{
F.J. Cabrerizo \\ University of Granada \\ cabrerizo@decsai.ugr.es
}

\author{
E. Herrera-Viedma \\ University of Granada \\ viedma@decsai.ugr.es
}

\begin{abstract}
Sometimes, the consensus reaching process in group decision making problems is a challenging task for the people who are in charge of the final choice (usually called experts). Firstly, the consensus is defined as a convergent and iterative process. This implies that it is necessary to keep the experts' attention during the whole process, even if it is longer than expected. Secondly, some of the experts tend to be rigid and they do not change their minds to help in the negotiation process easily. In such a way, we propose a new feedback mechanism that uses some gamification rules, designed as a reward distribution system, in order to transform that task into a game. This change can improve the consensus reaching process in both situations: keeping the experts' attention on the process and motivating those experts that should adjust their preferences.
\end{abstract}

\section{Introduction}

Solving group decision making problems has always been an important task for the society. There are some decisions that have to be made by a group of people (usually called experts) instead of a single person [1]. In such cases, if the motivations and interests of the group members are not the same, it is necessary to establish some rules to make a fair decision. Brexit is a clear example of this [2]. Here, the British Parliament, which is composed of 650 members having diverging positions over Brexit, must choose the best option among several ones such as the so-called Common Market 2.0 idea, staying in the single market, staying in the customs union, a no-deal Brexit, or Theresa May's withdrawal agreement [3].

In order to model the establishment of these rules, several methods have been proposed from a directive to a participatory range. Directive methods, as individual dominance or minority influence, let one person or a small group the control to make the decision for the rest of the people. On the other hand, participatory methods, as majority rules or consensus reaching processes [4], usually involve all the group members to implement a voting system or a negotiation procedure.

In this research, we are focused on the last method, the consensus reaching process [4]. Usually, consensus is defined as a convergent and iterative process to narrow the people's opinions. That is, a negotiation process to make a consensual decision. In such a way, the challenge has been the way to measure the consensus level. Before 70s, consensus was considered as a crisp measure, that is, total consensus if all the experts have exactly the same opinions, and non-consensus otherwise. However, with the use of fuzzy set theory [5], the term soft consensus emerged to model partial consensus scenarios [6]. In this way, if the experts express their preferences by means of the fuzzy set theory, it should be possible to measure the distance between their individual opinions, and compute a soft consensus measure to monitor the consensus state.

To manage these soft consensus reaching processes, the problem is modeled as a group of two or more experts, $E=\left\{e_{1}, e_{2}, \ldots, e_{m}\right\},(m \geq 2)$, and a solution set of possible alternatives or options, $X=$ $\left\{x_{1}, x_{2}, \ldots, x_{n}\right\},(n \geq 2)$. Then, the experts have to express their preferences and negotiate until they reach a high enough consensus level. To do so, a system usually acts as a moderator, computing every distance measure, monitoring the process, and showing to the experts the best way to converge with their preferences by sending them some feedback information.

These consensus models are considered dynamic by definition, because they are composed of several rounds or iterations. However, some recent contributions have gone one step further considering as dynamic each component of the model [7]: dynamic experts, dynamic alternatives, or dynamic preferences, for example. Even so, some adaptive models consider dynamic ways to compute the problem measures depending on the current state of the consensus process [8, 9, 10]. Usually, this state has been used to customize the feedback information received by the experts. 
One of the main drawbacks of the current consensus reaching models is the non-cooperative behaviour management. Occasionally, some experts with a different opinion from the majority preferences try to hinder the agreement. If they do not consider the feedback provided by the system, the consensus process will not converge and the consensus level will never be enough. Some authors have proposed new approaches to deal with this situation under specific environments [11], but there is still a research gap in the field.

A gamification-based approach may be useful in such settings. Gamification can be defined as the application of game mechanics outside the entertainment context to solve problems or engage users [12]. A possible example is to give the participant some kind of reward (such as points, badges, or even virtual money) [13]. In fact, the goal of the agents in a dynamic setting can be modified through this kind of rewards [14], and new economic models, based in game theory, can be applied.

Under this scenario, the main objective of this research is to present a new adaptive feedback mechanism, which uses some gamification rules implemented as a reward distribution system. In such a way, it can be used not only to keep the experts' attention during the whole process, but also to motivate those experts that should adjust their preferences to cooperate in the negotiation process in order to reach a consensus level as high as possible.

To do that, the document is organized in the following way. Section 2 introduces some basic aspects about consensus reaching processes for group decision making, non-cooperative expert's behaviour and gamification rules. Section 3 describe the feedback mechanism that we propose, including the new reward distribution system. Section 4 shows an illustrative case of use in a real world example. Finally, section 5 points out some concluding remarks about the novelties and performance of the proposal.

\section{Preliminaries}

In this section, some context information about group decision making problems is presented. Section 2.1 introduces the classic consensus reaching process for group decision making. Section 2.2 deals with the non-cooperative experts' behaviour. Finally, section 2.3 shows how the use of gamification rules could help in consensus reaching process modelling.

\section{Consensus Reaching Process}

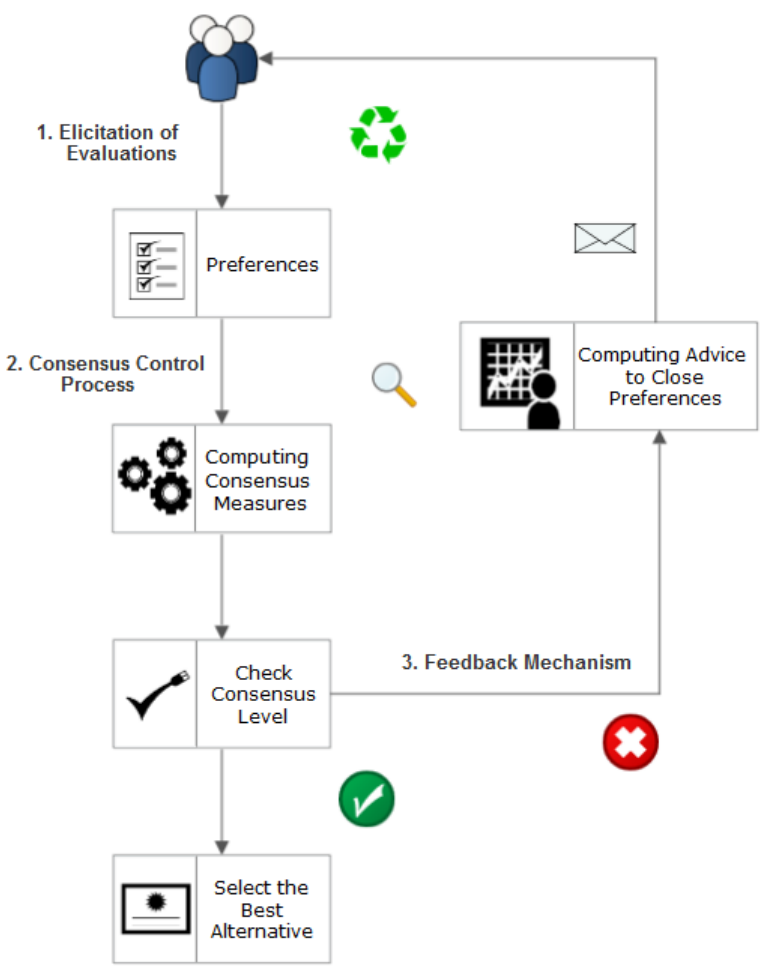

Figure 1. A consensus reaching process for group decision making.

\subsection{Consensus reaching processes for group decision making}

Consensus reaching processes for group decision making are composed of three steps (see Figure 1):

1. Elicitation of evaluations.

2. Consensus control process.

3. Feedback mechanism.

In the following three sub-subsections, we detail the aforementioned three steps.

2.1.1. Elicitation of evaluations Fuzzy preference relations are assumed to model the evaluations that the experts provide. They are used in consideration of their capacity to model decision processes and their ability to fuse individual evaluations into group ones [6].

Definition 1 A fuzzy preference relation $P$ on a finite set $X$ is a fuzzy set on the Cartesian product $X \times X$, that is, it is determined by means of a membership function $\mu_{P}: X \times X \rightarrow[0,1]$ over the set $X \times X$. 
The $n \times n$ matrix $P=\left(p_{i j}\right)$ is habitually used to characterize a fuzzy preference relation $P$, being the degree in which the option $x_{i}$ is preferred to the option $x_{j}$ represented by $p_{i j}=\mu_{P}\left(x_{i}, x_{j}\right)$. In this setting, the indifference between both options $\left(x_{i} \sim x_{j}\right)$ is represented by $p_{i j}=0.5$. If the option $x_{i}$ is entirely preferred to the option $x_{j}$, it is represented by $p_{i j}=1$, whereas if the option $x_{i}$ is preferred to the option $x_{j}$ $\left(x_{i} \succ x_{j}\right)$, it is represented by $p_{i j}>0.5$. In addition, as the elements of the main diagonal, $p_{i i}$, are not important in such a context, we write them as ' - ' [6].

2.1.2. Consensus control process The consensus achieved among the experts must be calculated once all of them have provided their evaluations. This is done by following a strategy habitually used when the experts utilize fuzzy preference relations that allow to calculate three consensus degrees. The strategy is as follows [9]:

- Definition of a similarity matrix, $S M^{k l}=$ $\left(s m_{i j}^{k l}\right)$, for each pair of experts $\left(e^{k}, e^{l}\right)$ as follows:

$$
s m_{i j}^{k l}=1-\left|p_{i j}^{k}-p_{i j}^{l}\right|
$$

- Aggregation of the similarity matrices to determine a consensus matrix, $C M=\left(\mathrm{cm}_{i j}\right)$. This is done by using the arithmetic mean as aggregation function $\phi$ :

$$
c m_{i j}=\phi\left(s m_{i j}^{k l}\right)
$$

- Calculation of three consensus degrees:

1. Consensus degree on pairs of options, $c p_{i j}$. This consensus degree represents the agreement reached on the pair formed by the options $x_{i}$ and $x_{j}$. It is characterized by the entry $(i, j)$ of $C M$ :

$$
c p_{i j}=c m_{i j}
$$

2. Consensus degree on options, $c a_{i}$. This consensus degree represents the agreement reached on the option $x_{i}$. Next expression calculates it:

$$
c a_{i}=\frac{\sum_{j=1 ; j \neq i}^{n}\left(c p_{i j}+c p_{j i}\right)}{2(n-1)}
$$

3. Consensus degree on the relation, $c r$. This consensus degree represents the total agreement reached. Next expression calculates it:

$$
c r=\frac{\sum_{i=1}^{n} c a_{i}}{n}
$$

The consensus reaching process is controlled by the consensus degree on the relation, $\mathrm{cr}$. This is done by contrasting the value of $c r$ with a threshold, $\alpha \in[0,1]$ [8]. When $c r \geq \alpha$, we apply a selection process that allows us to choose the best option for solving the group decision making problem [15]. Otherwise, a feedback mechanism is carried out with the aim of adjusting the opinions expressed by the experts. The objective of this is to increase $\mathrm{cr}$. Furthermore, a maximum number of rounds is habitually established to avoid the situation in which the consensus degree on the relation does not converge to $\alpha$ [16]. When this occurs, that is, the current round is higher than the maximum number of rounds, we must also apply the selection process [15].

2.1.3. Feedback mechanism As it has been noted, if the obtained $\mathrm{cr}$ is lower than the established threshold, a new round should start. However, before giving to the experts the opportunity to change their minds in order to modify the collective preference with their new opinions, a feedback mechanism must be implemented.

The main objective of this feedback mechanism is to automatically simulate the task carried out by a moderator in a real consensus reaching scenario. That is, it has to show to the experts the best way to narrow their preferences from an impartial point of view. In this way, it is necessary to compute other consensus measures, called proximity measures, as the distance from each individual opinion to the collective one [4].

Once the system has computed these proximity measures, the challenge is to find the controversial preferences and send to the experts the direction in which they should change their minds if they want to make their positions converge, improving the consensus degree on the relation and making an agreement with a particular solution.

\subsection{Non-cooperative experts' behaviour}

In this subsection, we are going to summarize the recent state of the art about non-cooperative behavior management in consensus reaching processes. It is important to note that non-cooperative experts are those who do not change their preferences, even if the moderator or the system itself ask them to do it for the sake (convergence) of the process.

The first approach about consensus strategies showing the differences between cooperative and non-cooperative behavior was proposed by Sheng-Yuan et al. in 2012 [17]. In 2013, Palomares et al. [11] started a new research line in the field. In their first contribution, the authors presented a fuzzy clustering approach to 
detect coalitions and non-cooperative behaviors. In such a way, the identification of non-cooperative experts was established as the very first step of the problem solving.

In 2015, Xuan-hua et al. [18] applied the previous approach to multi-criteria large group emergency decision scenarios. Moreover, the authors presented a method to automatically characterize two important parameters of a consensus reaching process: the maximum number of rounds and the minimum threshold for the achieved consensus level.

In 2016, Dong et al. [19] developed a new approach to deal with non-cooperative behaviors. The novelty of this method is the dynamic generation of the experts' weights. In this paper, the authors consider that in Palomares et al.'s approaches the management of this kind of behaviors is excessively dependent on a moderator and extremely demanding for him or her.

Finally, in 2018, some authors of previous papers joined their efforts to present a new approach based on social network analysis that manages the non-cooperative behaviors in these environments [20].

\subsection{Gamification}

This subsection is devoted to explain the concept of "gamification" and how the proposed method can take advantage of game theory to increase the performance of our feedback mechanism.

There is no exact definition for the term gamification, as its use overlaps between many fields of research [21]. Some authors define it as the extrapolation of game mechanics out of the context of entertainment to engage the user and solve problems [12]. In fact, gamification has been successfully applied in different fields, such as education, innovation, health, or marketing, among many others, for example, using gamification platforms that provide reward features in the form of points, reputation, coupons, levels, badges, or credits [13].

On the other hand, gamification proposes to use game playing theories in various contexts to solve difficult problems. For example, by adding a human-in-the-loop in a machine learning method it is possible to increase its performance. In [22], the authors improved the results of an ant colony algorithm by introducing humans playing a game into the process.

However, gamification is still an understudied area in the context of decision systems [12]. Some examples of the usefulness of its application may be in the field of personnel selection. In [12], it was used to obtain the characterization of the aspirant, analyzing the decisions he or she makes playing a game, in conjunction with a set of fuzzy rules. Related to this, in [13], the authors proposed the decision criteria and weights for deciding which (commercial) gamification platform should be considered. These criteria include the competitiveness of the product (sales, architecture, function, performance), or the continuity of the service (vendor stability, contract terms), among others. The authors indicated that these weights can help to understand what aspects should be considered for a successful gamification.

A gamified effort feedback mechanism was proposed in [23] to make decision support systems easier to understand and used by non-experts, and to drive decision makers to invest more effort into the decision making process. Results demonstrated that the effects of that feedback mechanism not only increased motivation in humans, but that these effects are more pronounced if the feedback is provided during each decision task (and not after it). Moreover, the egoistic behavior of agents in an interactive setting can be influenced on the basis of incentives to modify the goals of those agents, through the use of game theory, to model new economic interactions [14].

With regard to the use of rewards, it is in one of the branches of machine learning, the reinforcement learning, where they can have more relevance [24]. This approach is based on how subjects make decisions through rewards, positive or negative. The goal is to maximize the action in the environment that produces the most reward, but not the result of the action itself. Examples of these methods may be the Monte Carlo Tree Search methods, or the Q-learning algorithm. However, the agent cannot spend this reward, for example, to perform an action more exploratory (or risky) than exploitative.

The proposal presented in this paper takes ideas from the approaches explained above. On the one hand, the concept of reward can be seen as a payoff for performing an action, but, on the other hand, it can be seen as gamification reward features (such as virtual money, reputation or coupons), which can be spent later to add more weight to a decision. In fact, different economic models could be applied, such as Commodity Market model or Bargaining model, in which providers and consumers of a service can negotiate among themselves to meet their objectives until they agree on a price [25].

\section{A new feedback mechanism to increase the experts' experience using gamification tools}

Here, we describe our proposal for a new feedback mechanism based on game theory, which is structured into three steps (see Figure 2): 


\section{Feedback Mechanism}

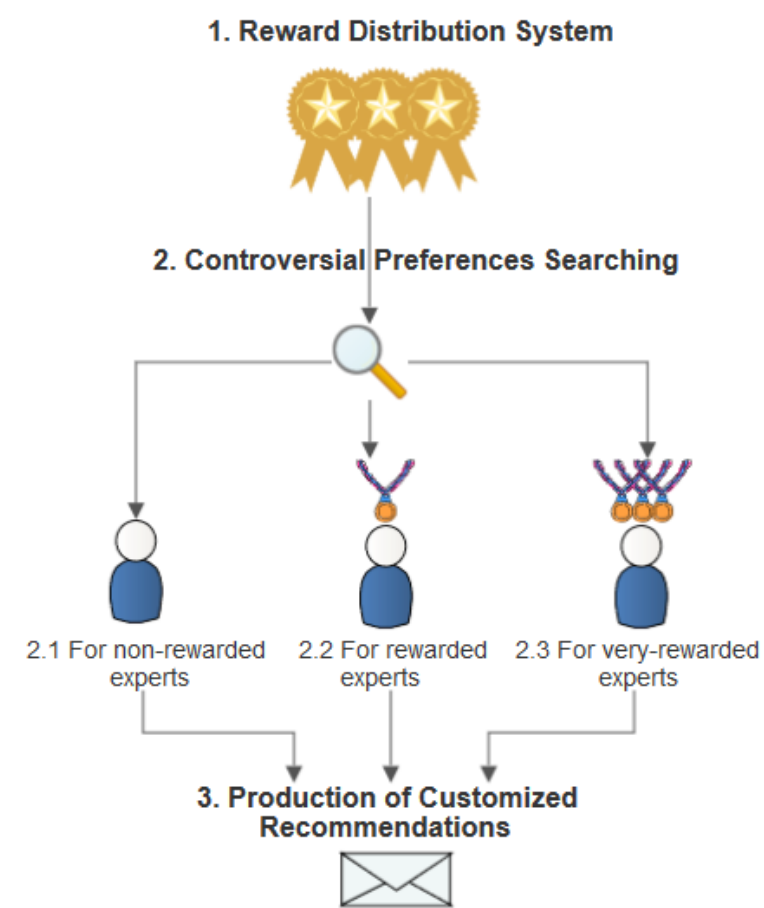

Figure 2. Feedback mechanism based on game theory.

1. Reward distribution system.

2. Controversial preferences searching.

3. Production of customized recommendations.

\subsection{Reward distribution system}

This system has been designed based on the assumption that if the experts with a cooperative behaviour (those that follow the advice provided by the system in the previous stage) receive a kind of reward that can be turned into some benefits in the next stage, the consensus reaching task will be seen as a kind of game by the experts. As gamification studies have demonstrated (see Section 2.3), not only all the experts will keep their attention focused on the problem but also those with non-cooperative behaviour would be willing to change their opinions to obtain the promised benefits.

In this particular case, the obtained rewards are turned into a double benefit:

- To increase the individual weight in the aggregation function that computes the collective preference (see Section 3.2.1).
- To receive a fewer amount of recommendations to change the individual preferences in the next consensus round (see Section 3.2.2).

To accomplish this task, the reward distribution system is structured into the following three steps:

1. If round $=1 \rightarrow$ for each expert $e^{k}$ a reward $R e w^{k}=0$.

2. If round $>1 \rightarrow$ for each expert $e^{k}$ a reward $R e w^{k}=R e w^{k}+1$ is assigned for each preference changed according to the recommendations received in the previous round.

3. To normalize the reward vector Rew in order to manage weights in the interval $[0,1]$ in the following way:

$$
N R e w^{k}=R e w^{k} / \max (R e w)
$$

After the reward distribution, each expert is included according to his or her reward degree into one of these three subsets:

- Very rewarded, $E_{v r}$.

- Rewarded, $E_{r}$.

- Non-rewarded, $E_{n r}$.

The normalized reward vector NRew of each expert is interpreted as a fuzzy subset. That is, $N R e w^{k} \in$ $[0,1]$ symbolizes the reward degree assigned to the expert $e^{k}$. A fuzzy matching mechanism is then carried out to include each expert into one of the subsets (the parameters associated with the fuzzy matching mechanism depend on the problem at hand). Each subset of experts is a fuzzy set determined by a membership function. In addition, two membership thresholds, $\beta_{1}$ and $\beta_{2}$, are determined to distinguish these three subsets.

\subsection{Controversial preferences searching}

To discover the controversial preferences (those with low agreement), the first step consist in computing some proximity measures. Secondly, we need to define the searching strategy.

3.2.1. Computing proximity measures First, our feedback mechanism computes the agreement achieved between the individual evaluations and the group ones. This is done by following the strategy encountered in most of the existing approaches [4]: 
- A collective matrix, $P^{c}=\left(p_{i j}^{c}\right)$, is determined by aggregating the fuzzy preference relations given by the experts:

$$
p_{i j}^{c}=\Phi\left(p_{i j}^{1}, p_{i j}^{2}, \ldots, p_{i j}^{m}\right)
$$

As the experts have different reward degrees, the aggregation function, $\Phi$, must take into account it. When weighted values are aggregated, the procedure used consists usually in transform the values under the weight to generate a new value. The new values are then aggregated by means of an aggregation function. In this research, the weighted average is utilized to take into account the reward degrees, which act as weights of the evaluations:

$$
p_{i j}^{c}=\frac{\sum_{k=1}^{m} N R e w^{k} \cdot p_{i j}^{k}}{\sum_{k=1}^{m} N R e w^{k}}
$$

- Using the collective matrix, the proximity measures are computed as follows:

1. Proximity measure on pairs of options, $p p_{i j}^{k}$. It determines the similarity between the evaluation expressed by the expert $e^{k}$ on a pair of options $\left(x_{i}, x_{j}\right)$ and the collective one:

$$
p p_{i j}^{k}=1-\left|p_{i j}^{k}-p_{i j}^{c}\right|
$$

2. Proximity measure on options, $p a_{i}^{k}$. It determines the similarity between the evaluation expressed by the expert $e^{k}$ on the option $x_{i}$ and the collective one:

$$
p a_{i}^{k}=\frac{\sum_{j=1, j \neq i}^{n}\left(p p_{i j}^{k}+p p_{j i}^{k}\right)}{2(n-1)}
$$

3. Proximity measure on the relation, $p r^{k}$. It determines the global similarity between the evaluations provided by the expert $e^{k}$ and the collective one:

$$
p r^{k}=\frac{\sum_{i=1}^{n} p a_{i}^{k}}{n}
$$

3.2.2. Searching strategies Second, our feedback mechanism identifies the preferences to be adjusted with the purpose of improving the agreement in the next round. This is done by using three strategies that determine the preferences that the experts must adjust. In other words, a different strategy is entailed by each one of the previous subsets to discover the controversial preferences.
1. Searching controversial preferences for non-rewarded experts. Based on common sense, this subset includes experts whose behaviour is non-cooperative. To penalize this attitude, more modifications should be suggested here. As a consequence, this subset follows a strategy trying to adjust the evaluations on all the pairs of options having a low consensus. This is done in the following way:

(a) The mean of all consensus degrees on pair of options determines a threshold, $\gamma_{1}$ :

$$
\gamma_{1}=\sum_{i=1}^{n}\left(\sum_{j=1, j \neq i}^{n} c p_{i j}\right) /\left(n^{2}-n\right)
$$

(b) Identification of the pairs of options, $P O$, having a consensus degree smaller than $\gamma_{1}$ :

$$
P O=\left\{\left(x_{i}, x_{j}\right) \mid c p_{i j}<\gamma_{1}\right\}
$$

(c) Identification of the controversial evaluations, $C E_{n r}^{k}$, that each expert $e^{k} \in E_{n r}$ must modify:

$$
C E_{n r}^{k}=P O
$$

2. Searching controversial preferences for rewarded experts. It seems logical that the subset of rewarded experts applies a strategy that reduces the number of evaluations to be adjusted. This is done by analyzing the agreement from the perspective of the options instead of all the pairs of options in disagreement. In other words, this strategy only takes into account the evaluations on the options having a low agreement. Furthermore, for the identified options in disagreement, this strategy requires that only the experts having a proximity measure at the level of options smaller than a proximity threshold $\delta_{1}$ adjust their evaluations, whereas in the previous strategy all the experts are suggested to adjust the identified evaluations. For each expert, $e^{k} \in E_{r}$, this is done as follows:

(a) The mean of the consensus degrees on options determines a threshold, $\gamma_{2}$ :

$$
\gamma_{2}=\sum_{i=1}^{n} c a_{i} / n
$$

(b) Identification of the options, $O$, to be adjusted:

$$
O=\left\{x_{i} \mid c a_{i}<\gamma_{2}\right\}
$$


(c) Identification the pairs of options, $P O$, to be adjusted:

$$
P O=\left\{\left(x_{i}, x_{j}\right) \mid x_{i} \in O \wedge c p_{i j}<\gamma_{1}\right\}
$$

(d) For each option $x_{i} \in O$, we determine a threshold, $\delta_{1}$, as follows:

$$
\delta_{1}=\sum_{k=1}^{m} p a_{i}^{k} / m, e^{k} \in E_{r}
$$

(e) Identification of the controversial evaluations, $C E_{r}^{k}$, that must be adjusted:

$$
C E_{r}^{k}=\left\{\left(x_{i}, x_{j}\right) \in P O \mid p a_{i}^{k}<\delta_{1}\right\}
$$

3. Searching controversial preferences for very rewarded experts. The subset $E_{v r}$ is composed of the experts having a high reward degree. Therefore, these experts do not need to adjust excessively their opinions to get a good evaluation (it is considered that they have already changed their mind in the right direction). As a consequence, the strategy applied here should suggest fewer changes than the previous two strategies to improve the consensus. Concretely, for the pairs of options identified in disagreement, this strategy only changes the evaluations provided by the experts having a proximity measure smaller than a proximity threshold at the level of pair of options, $\delta_{2}$. For each expert, $e^{k} \in E_{v r}$, this is done as follows:

(a) Identification of the options, $O$, to be adjusted:

$$
O=\left\{x_{i} \mid c a_{i}<\gamma_{2}\right\}
$$

(b) Identification of the pairs of options, $P O$, to be adjusted:

$$
P O=\left\{\left(x_{i}, x_{j}\right) \mid x_{i} \in O \wedge c p_{i j}<\gamma_{1}\right\}
$$

(c) For each pair of options $\left(x_{i}, x_{j}\right) \in P O$, we determine a threshold $\delta_{2}$, as follows:

$$
\delta_{2}=\sum_{k=1}^{m} p p_{i j}^{k} / m, e^{k} \in E_{v r}
$$

(d) Identification of the controversial evaluations, $C E_{v r}^{k}$, that must be adjusted:

$$
\begin{gathered}
C E_{v r}^{k}=\left\{\left(x_{i}, x_{j}\right) \in P O \mid p a_{i}^{k}<\delta_{1} \wedge\right. \\
\left.\wedge p p_{i j}^{k}<\delta_{2}\right\}
\end{gathered}
$$

\subsection{Advising}

Once the feedback mechanism identifies the controversial evaluations, the next step consists in indicating the right direction of the changes. As it is done in other methods [8], our feedback mechanism uses two direction rules. In such a way, for each evaluation recognized as controversial, the feedback mechanism introduced in this research proposes the adjustment of the current evaluation as follows:

- The evaluation expressed by the expert $e^{k}$ on the pair $x_{i}$ and $x_{j}$ have to be increased if $p_{i j}^{k}<p_{i j}^{c}$.

- The evaluation expressed by the expert $e^{k}$ on the pair $x_{i}$ and $x_{j}$ have to be decreased if $p_{i j}^{k}>p_{i j}^{c}$.

In practice, the feedback mechanism proposed the adjustment of the current evaluations by means of two direction rules: (i) the symbol + means that this evaluation have to be increased, and (ii) the symbol means that this evaluation have to be decreased.

In summary, experts with a high proximity to the collective solution or experts very rewarded because they have already changed their preferences according to the system recommendations in previous rounds should receive a light recommendation scheme, whereas distant experts from the collective solution with a non-cooperative behaviour will receive a harder recommendation.

\section{Case of use}

In order to validate the concepts that we have introduced in this research, we present a case of use of the model. Let us suppose that a city (or a country) government wants to invest some money by improving some of its public services, which are: $x_{1}=$ cleaning services, $x_{2}=$ health services, $x_{3}=$ education services, and $x_{4}=$ security services. The idea is to distribute the funding among the public services depending on the necessities of each one of them. To accomplish this task, four experts are inquired.

To solve this particular problem, we use the proposed model. As there are to many intermediate calculations and some space restrictions, firstly, we just focus on a single intermediate consensus round to show each step of the process. Then, we analyze the convergence of the model by simulating different scenarios.

The proposed case of use is set up as follows: the consensus threshold $\alpha=0.75$, the membership thresholds (related to the rewards received), $\beta_{1}$ and $\beta_{2}$ are 0.65 and 0.45 , respectively. Finally, the maximum number of consensus rounds is 6 . 


\subsection{Consensus reaching process}

4.1.1. Elicitation of evaluations In the first discussion round, experts provide their initial preference relations, but in the rest of rounds, they should change them by following the system recommendations. We present an intermediate round in which the experts have previously received some advice. In this situation, the experts provide their particular opinions by using the following fuzzy preference relations:

$$
\begin{aligned}
P^{1} & =\left(\begin{array}{cccc}
- & 0.8 & 0.8 & 0.8 \\
0.2 & - & 0.6 & 0.7 \\
0.3 & 0.4 & - & 0.6 \\
0.3 & 0.3 & 0.4 & -
\end{array}\right) P^{2}=\left(\begin{array}{cccc}
- & 0.0 & 0.2 & 0.2 \\
1.0 & - & 0.7 & 0.8 \\
0.8 & 0.3 & - & 0.4 \\
0.9 & 0.2 & 0.6 & -
\end{array}\right) \\
P^{3} & =\left(\begin{array}{cccc}
- & 0.4 & 0.0 & 0.2 \\
0.6 & - & 0.1 & 0.1 \\
1.0 & 0.9 & - & 0.4 \\
0.9 & 1.0 & 0.6 & -
\end{array}\right) P^{4}=\left(\begin{array}{cccc}
- & 0.4 & 0.2 & 0.2 \\
0.6 & - & 0.1 & 0.1 \\
0.9 & 0.9 & - & 0.4 \\
0.8 & 0.8 & 0.6 & -
\end{array}\right)
\end{aligned}
$$

4.1.2. Consensus control process The first step is the computation of one similarity matrices for each pair of experts (they are omitted). Then, we calculate the consensus matrix $C M$, whose values can be used to generate the consensus degrees at different levels:

1. Consensus degrees on pairs of options:

$$
C M=\left(\begin{array}{cccc}
- & 0.60 & 0.60 & 0.70 \\
0.60 & - & 0.62 & 0.55 \\
0.63 & 0.62 & - & 1.00 \\
0.68 & 0.52 & 1.00 & -
\end{array}\right)
$$

2. Consensus degree on options:

$$
c a_{1}=0.64 \quad c a_{2}=0.58 \quad c a_{3}=0.75 \quad c a_{4}=0.74
$$

3. Consensus degree on the relation:

$$
c r=0.68
$$

As $c r=0.68$ is lower than the established threshold ( $\alpha=0.75$ ), and the current consensus round is not the last one, the system starts the feedback mechanism.

\subsubsection{Feedback mechanism}

- Rewards distribution system: During the second and next consensus rounds, if the feedback mechanism is activated, the reward distribution system assigns a reward to those experts that have followed the received recommendations (see
Section 3.1). The final and normalized reward values obtained by using (6) are:

$$
\begin{aligned}
& N R e w^{1}=0.70 \quad N R e w^{2}=0.50 \\
& N \text { Rew }^{3}=0.40 \quad N R e w^{4}=0.40
\end{aligned}
$$

To compute the customized advice in a proper way, the system takes into account the reward received by each expert. Thus, they are included into three different subsets (taking $\beta_{1}$ and $\beta_{2}$ as reference):

$$
E_{n r}=\left\{e^{3}, e^{4}\right\} \quad E_{r}=\left\{e^{2}\right\} \quad E_{v r}=\left\{e^{1}\right\}
$$

- Computing proximity measures: In the same way that we have computed the consensus degree at three different levels, we compute the proximity measures. First, the collective fuzzy preference relation is calculated by aggregating the four individual fuzzy preference relations using (8). Note that the rewards obtained in the previous step, are used here. Then, $\mathrm{P}$ proximity measures on pairs of options (9), proximity measures on options (10) and proximity measures on the relation (11) are obtained. These values are omitted due to space restrictions.

\section{- Identifying for controversial evaluations:}

1. Identifying controversial evaluations for non-rewarded experts:

(a) The threshold $\gamma_{1}=0.68$.

(b) Pairs of options with a consensus degree lower than $\gamma_{1}$ :

$$
\begin{gathered}
P O=\left\{\left(x_{1}, x_{2}\right),\left(x_{1}, x_{3}\right),\left(x_{2}, x_{1}\right),\right. \\
\left(x_{2}, x_{3}\right),\left(x_{2}, x_{4}\right),\left(x_{3}, x_{1}\right), \\
\left.\left(x_{3}, x_{2}\right),\left(x_{4}, x_{2}\right)\right\}
\end{gathered}
$$

(c) Evaluations that should be modified by experts $e^{3}$ and $e^{4}$ :

$$
\begin{aligned}
& C E_{h c}^{3}=P O \\
& C E_{h c}^{4}=P O
\end{aligned}
$$

2. Identifying controversial evaluations for rewarded experts:

(a) The threshold $\gamma_{2}=0.68$.

(b) Options whose consensus degree should be higher:

$$
O=\left\{x_{1}, x_{2}\right\}
$$


(c) Pair of options in disagreement:

$$
\begin{aligned}
P O=\{ & \left(x_{1}, x_{2}\right),\left(x_{1}, x_{3}\right),\left(x_{2}, x_{1}\right), \\
& \left.\left(x_{2}, x_{3}\right),\left(x_{2}, x_{4}\right)\right\}
\end{aligned}
$$

(d) The threshold $\delta_{1}=0.75$ for the option $x_{1}$ and 0.73 for the option $x_{2}$.

(e) Evaluations that should be modified by the expert $e^{2}$ :

$$
\begin{aligned}
C E_{c}^{2}=\left\{\left(x_{1}, x_{2}\right),\left(x_{1}, x_{3}\right),\left(x_{2}, x_{1}\right),\right. \\
\\
\left.\left(x_{2}, x_{3}\right),\left(x_{2}, x_{4}\right)\right\}
\end{aligned}
$$

3. Identifying controversial evaluations for very rewarded experts:

(a) Options with a consensus degree lower than expected:

$$
O=\left\{x_{1}, x_{2}\right\}
$$

(b) Pair of options in disagreement:

$$
\begin{aligned}
P O=\{ & \left(x_{1}, x_{2}\right),\left(x_{1}, x_{3}\right),\left(x_{2}, x_{1}\right), \\
& \left.\left(x_{2}, x_{3}\right),\left(x_{2}, x_{4}\right)\right\}
\end{aligned}
$$

(c) The average of the proximity measures on pairs of options previously identified is the threshold $\delta_{2}$.

(d) Evaluations to be modified by the expert $e^{1}$ :

$$
C E_{h c}^{1}=\left\{\left(x_{1}, x_{2}\right),\left(x_{1}, x_{3}\right)\right\}
$$

- Advising: The feedback mechanism uses the computed proximity measures to suggest the modification of the current evaluations (see Section 3.3). According to the direction rules, the recommendations + mean that this expert should increase these evaluations. On the other hand, the recommendations - mean that this expert should decrease these evaluations.

$$
\begin{gathered}
P^{1}=\left(\begin{array}{llll}
= & - & = \\
= & = & = \\
= & = & =
\end{array}\right) P^{2}=\left(\begin{array}{llll}
- & + & = \\
- & & - & - \\
= & = & = \\
= & = & =
\end{array}\right) \\
P^{3}=\left(\begin{array}{lll}
- & + & = \\
- & + & + \\
= & - & =
\end{array}\right) P^{4}=\left(\begin{array}{lll}
+ & + & = \\
- & + & + \\
- & - & =
\end{array}\right)
\end{gathered}
$$

\subsection{Convergence analysis}

In order to study the convergence of the model, we have simulated several group decision making problems with various sets of experts and options. If the experts accept the recommendations proposed by the system, we can observe that the consensus is growing up in each discussion round (see Figure 3). Hence, the model developed in this research allow to the experts to make decisions with higher consensus.

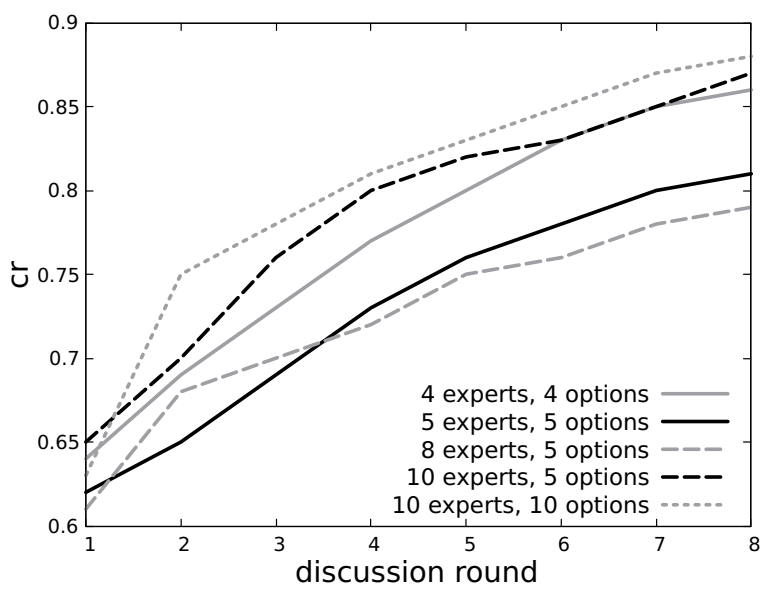

Figure 3. $c r$ in successive discussion rounds.

\section{Concluding remarks}

In this research, a new feedback mechanism using some gamification rules implemented as a reward distribution system has been presented.

We have transformed the consensus reaching task into a game for the experts by giving them some reward if they obey the recommendations. The system can be used not only to keep the experts' attention during the whole process but also to motivate those experts that should adjust their preferences to cooperate in the negotiation process in order to reach a consensus level as high as possible. To do this, the system implements three strategies to identify those preferences that should be modified by the experts if they want that the consensus increases. The strategies are performed depending on the experts' rewarding level by considering the different computed values (consensus degrees and proximity measures). The idea is that the opinions of the most rewarded experts do not have to be strongly changed (it is supposed that they have changed their preferences enough in the previous rounds).

As future work, we think that the application of mathematical rules of game theory to the current consensus reaching processes could lead the experts to make better decisions. 


\section{Acknowledgements}

The work was supported by the Grants from the FEDER funds provided by the Spanish Ministry of Economy and Competitiveness (Nos. TIN2016-75850-R, TIN2017-85727-C4-2-P) and by the Programa de Fomento e Impulso de la actividad investigadora de la Universidad de Cádiz.

\section{References}

[1] F. J. Cabrerizo, J. A. Morente-Molinera, W. Pedrycz, A. Taghavi, and E. Herrera-Viedma, "Granulating linguistic information in decision making under consensus and consistency," Expert Systems with Applications, vol. 99, pp. 83-92, 2018.

[2] N. Carl, J. Dennison, and G. Evans, "European but not European enough: An explanation for Brexit," European Union Politics, vol. 20, no. 2, pp. 282-304, 2019.

[3] S. Brakman, H. Garretsen, and T. Kohl, "Consequences of Brexit and options for a 'Global Britain'," Papers in Regional Science, vol. 97, no. 1, pp. 55-72, 2018.

[4] E. Herrera-Viedma, F. J. Cabrerizo, J. Kacprzyk, and W. Pedrycz, "A review of soft consensus models in a fuzzy environment," Information Fusion, vol. 17, pp. 4-13, 2014.

[5] L. Zadeh, "Fuzzy sets," Information and Control, vol. 8, no. 3, pp. 338-353, 1965.

[6] J. Kacprzyk, "Group decision making with a fuzzy linguistic majority," Fuzzy Sets and Systems, vol. 18, no. 2, pp. 105-118, 1986.

[7] I. J. Pérez, F. J. Cabrerizo, S. Alonso, Y. C. Dong, F. Chiclana, and E. Herrera-Viedma, "On dynamic consensus processes in group decision making problems," Information Sciences, vol. 459, pp. 20-35, 2018.

[8] F. Mata, L. Martínez, and E. Herrera-Viedma, "An adaptive consensus support model for group decision-making problems in a multigranular fuzzy linguistic context," IEEE Transactions on Fuzzy Systems, vol. 17, no. 2, pp. 279-290, 2009.

[9] I. J. Pérez, F. J. Cabrerizo, S. Alonso, and E. Herrera-Viedma, "A new consensus model for group decision making problems with non homogeneous experts," IEEE Transactions on Systems, Man, and Cybernetics: Systems, vol. 44, no. 4, pp. 494-498, 2014.

[10] F. J. Cabrerizo, I. J. Pérez, J. A. Morente-Molinera, S. Alonso, and E. Herrera-Viedma, "An adaptive feedback mechanism for consensus reaching processes based on individuals' credibility," in Proceedings of the 52nd Hawaii International Conference on System Sciences, pp. 1678-1687, 2019.

[11] I. Palomares, L. Martínez, and F. Herrera, "Fuzzy clustering approach for non-cooperative behavior detection in consensus reaching processes," in Proceedings of the Fourth International Workshop on Knowledge Discovery, Knowledge Management and Decision Support, pp. 37-44, 2013.

[12] J. Albadán, P. Gaona, C. Montenegro, R. González-Crespo, and E. Herrera-Viedma, "Fuzzy logic models for non-programmed decision-making in personnel selection processes based on gamification," Informatica, vol. 29, no. 1, pp. 1-20, 2018.
[13] S. Kim, "Decision support model for introduction of gamification solution using AHP," The Scientific World Journal, vol. 2014, article ID 714239, 2014.

[14] I. C. Konstantakopoulos, A. R. Barkan, S. He, T. Veeravalli, H. Liu, and C. Spanos, "A deep learning and gamification approach to improving human-building interaction and energy efficiency in smart infrastructure," Applied Energy, vol. 237, pp. 810-821, 2019.

[15] F. J. Cabrerizo, R. Heradio, I. J. Pérez, and E. Herrera-Viedma, "A selection process based on additive consistency to deal with incomplete fuzzy linguistic information," Journal of Universal Computer Science, vol. 16, no. 1, pp. 62-81, 2010.

[16] E. Herrera-Viedma, S. Alonso, F. Chiclana, and F. Herrera, "A consensus model for group decision making with incomplete fuzzy preference relations," IEEE Transactions on Fuzzy Systems, vol. 15, no. 5, pp. 863-877, 2007.

[17] S.-Y. Tu and A. H. Sayed, "Diffusion strategies outperform consensus strategies for distributed estimation over adaptive networks," IEEE Transactions on Signal Processing, vol. 60, no. 12, pp. 6217-6234, 2012.

[18] X.-H. Xu, Z.-J. Du, and X.-H. Chen, "Consensus model for multi-criteria large-group emergency decision making considering non-cooperative behaviors and minority opinions," Decision Support Systems, vol. 79, pp. 150-160, 2015.

[19] Y. Dong, H. Zhang, and E. Herrera-Viedma, "Integrating experts' weights generated dynamically into the consensus reaching process and its applications in managing non-cooperative behaviors," Decision Support Systems, vol. 84, pp. 1-15, 2016.

[20] H. Zhang, I. Palomares, Y. Dong, and W. Wang, "Managing non-cooperative behaviors in consensus-based multiple attribute group decision making: An approach based on social network analysis," Knowledge-Based Systems, vol. 162, pp. 29-45, 2018.

[21] S. Deterding, D. Dixon, R. Khaled, and L. Nacke, "From game design elements to gamefulness: Defining 'gamification',' in Proceedings of the 15th International Academic MindTrek Conference: Envisioning Future Media Environments, pp. 9-15, ACM, 2011.

[22] A. Holzinger, M. Plass, M. Kickmeier-Rust, K. Holzinger, G. C. Crişan, C.-M. Pintea, and V. Palade, "Interactive machine learning: Experimental evidence for the human in the algorithmic loop," Applied Intelligence, vol. 49, no. 7, pp. 2401-2414, 2019.

[23] J. Frysak and E. W. N. Bernroider, "Digital gamified effort feedback mechanism to enhance information coverage in multi-attribute decision-making," in Proceedings of the 8th International Conference on Information, Intelligence, Systems \& Applications, pp. 1-6, IEEE, 2017.

[24] G. N. Yannakakis and J. Togelius, Playing Games, pp. 91-150. Cham: Springer International Publishing, 2018.

[25] P. Samimi and A. Patel, "Review of pricing models for grid \& cloud computing," in Proceedings of the 2011 IEEE Symposium on Computers \& Informatics, pp. 634-639, 2011. 